УДК 517.9

\title{
Weighted Fractional Neutral Functional Differential Equations
}

\author{
Mohammed S. Abdo* \\ Department of Mathematics \\ Dr. Babasaheb Ambedkar Marathwada University \\ Aurangabad-431 004 (M.S.) \\ India \\ Department of Mathematics \\ Hodeidah University \\ Al-Hodeidah-3114 \\ Yemen \\ Satish K. Panchal ${ }^{\dagger}$ \\ Department of Mathematics \\ Dr. Babasaheb Ambedkar Marathwada University \\ Aurangabad-431 004 (M.S.) \\ India
}

Received 19.12.2017, received in revised form 24.05.2018, accepted 16.07.2018

In this paper, we consider a weighted neutral functional differential equation of fractional order $0<\alpha<1$, with nonzero initial values, infinite delay, and the standard Riemann-Liouville fractional derivative. By using a variety of tools of fractional calculus including the Schauder fixed point theorem and the Banach fixed point theorem, we verify the existence, uniqueness and continuous dependence of solution of weighted neutral problem.

Keywords: fractional functional differential equations, fractional derivative and fractional integral, existence and continuous dependence, fixed point theorem.

DOI: 10.17516/1997-1397-2018-11-5-535-549.

\section{Introduction}

In the last few years, the fractional differential equations have attracted a considerable interest in mathematics and many applications and also played a very important role in mathematical modeling to systems and processes in various sciences such as physics, mechanics, chemistry, engineering, etc. For more details, see the monographs of Abbas et al. [1], Kilbas et al. [21], Miller and Ross [25], Podlubny [28] and Samko et al. [29], and the papers of Delboso and Rodino [9], Diethelm et al. [11,12], Gaul et al. [16], Glockle and Nonnenmacher [17], Metzler et al. [24], Momani et al. [26], Momani and Hadid [27], Yu and Gao [30] and the references therein.

Due to the application of fractional differential equations in many areas of applied mathematics, there has been a major development and an increasing interest in the investigation of the neutral functional differential equation with the effect of infinity delay. To our knowledge,

\footnotetext{
*msabdo1977@gmail.com

$\dagger$ drpanchalsk@gmail.com

(c) Siberian Federal University. All rights reserved
} 
fractional delay neutral functional differential equations has not been extensively studied. Especially, the results dealing with the weighted fractional differential equations are comparatively scarce. Among these works, see $[2-4,6,7,10,14]$. For example in [7], Benchohra et al., used the Banach fixed point theorem and the nonlinear alternative of Leray-Schauder to investigate the existence and uniqueness of solutions for the following fractional neutral functional differential equation with infinite delay

$$
\begin{aligned}
& D_{0}^{\alpha}\left[y(t)-g\left(t, y_{t}\right)\right]=f\left(t, y_{t}\right), \quad t \in[0, b], \\
& y(t)=\phi(t), \quad t \in(-\infty, 0],
\end{aligned}
$$

where $0<\alpha<1, D_{0}^{\alpha}$ is the standard Riemann-Liouville fractional derivative, $\phi \in \mathcal{B}$ with $\phi(0)=$ $0, \mathcal{B}$ is called a phase space and $f, g:[0, b] \times \mathcal{B} \longrightarrow \mathbb{R}(b>0)$ are given functions satisfy certain conditions with $g(0, \phi)=0$.

In [4], Agarwal et al. investigated the existence result of the following problem with finite delay

$$
\begin{aligned}
& { }^{c} D^{\alpha}\left[y(t)-g\left(t, y_{t}\right)\right]=f\left(t, y_{t}\right), \quad t \in\left[t_{0}, \infty\right), \\
& y_{t_{0}}=\phi \in \mathcal{C},
\end{aligned}
$$

where $0<\alpha<1,{ }^{c} D^{\alpha}$ is the Caputo fractional derivative, $f, g:\left[t_{0}, \infty\right) \times \mathcal{C} \longrightarrow \mathbb{R}^{n}$ are given functions satisfy some assumptions, and $\mathcal{C}=C\left([-\tau, 0], \mathbb{R}^{n}\right)(\tau>0)$ be the space of continuous functions on $[-\tau, 0]$. The results are proved by means of the Krasnoselskii fixed point theorem.

In [14], Dong considered a weighted fractional functional differential equation with infinite delay

$$
\begin{aligned}
& D_{0}^{\alpha} y(t)=f\left(t, \widetilde{y}_{t}\right), \quad t \in(0, b], \\
& \widetilde{y}_{0}=\phi \in \mathcal{B},
\end{aligned}
$$

where $0<\alpha \leqslant 1, D_{0}^{\alpha}$ is the standard Riemann-Liouville fractional derivative, $f:(0, b] \times \mathcal{B} \longrightarrow \mathbb{R}$ $(b>0)$ is given function satisfying some assumptions, $\widetilde{y}(t)=t^{1-\alpha} y(t)$ and $\mathcal{B}$ be the phase space. The author used the Banach contraction principle and Schauder fixed point theorem to obtain the existence and uniqueness results. Moreover he also discussed the continuous dependence of solutions for problem (3)-(4) in the space $C_{1-\alpha}((0, b])$.

Motivated by the above works, and inspired by [14], we consider a weighted fractional neutral functional differential equation with infinite delay

$$
\begin{aligned}
& D_{0}^{\alpha}\left[y(t)-g\left(t, \widetilde{y}_{t}\right)\right]=f\left(t, \widetilde{y}_{t}\right), \quad t \in(0, b] \\
& \widetilde{y}_{0}=\phi,
\end{aligned}
$$

where $0<\alpha \leqslant 1, D_{0}^{\alpha}$ is the Riemann-Liouville fractional derivative, $f, g:(0, b] \times \mathcal{B} \longrightarrow \mathbb{R}(b>0)$ satisfy certain conditions that will be specified later, $\mathcal{B}$ the phase space of functions mapping $(-\infty, 0]$ into $\mathbb{R}$, which will be specified in Section $2 ., \phi \in \mathcal{B}$ with $\widetilde{\phi}(t)=\phi(t)$ for $t \in(-\infty, 0]$ and $\widetilde{y}(t)=t^{1-\alpha} y(t)$.

The purpose of this paper is to establish and develope the existence, uniqueness and continuous dependence of solutions for the problem (5)-(6) with infinite delay and nonzero initial values by means of Schauder fixed point theorem and Banach fixed point theorem. The results obtained here generalizes the results of [14].

The paper is organized in five sections, In Section 2 we introduce some preliminaries and list the hypotheses that will be used throughout this paper. Section 3 is devoted to proving the 
existence and uniqueness of solutions of (5)-(6). Section 4 addressing the continuous dependence of solutions to such equations in the space $C_{1-\alpha}((0, b])$. Finally, the conclusion is given in Section 5 .

\section{Preliminaries}

In this section, some required notations, definitions and some hypotheses are given. Let $C((0, b])$ be the space of all continuous real functions defined on $(0, b]$ and $L_{l o c}^{1}(0, b]$ be the space of all real functions defined on $(0, b]$ which are locally lebesgue integrable. we also consider the space $C_{1-\alpha}((0, b], \mathbb{R})$ is the Banach space consisting of all continuous functions $\delta:(0, b] \rightarrow \mathbb{R}$ such that $\lim _{t \rightarrow 0} t^{1-\alpha} \delta(t)$ exists, endowed with the norm $\|\delta\|_{C_{1-\alpha}}=\sup \left\{\left|t^{1-\alpha} \delta(t)\right|: t \in(0, b]\right\}$.

For any function $y$ defined on $(-\infty, b]$ and any $t \in(0, b]$, we denote by $y_{t}$ the element of $\mathcal{B}$ defined by

$$
y_{t}(s)=y(t+s), \text { for }-\infty<s \leqslant 0 .
$$

We will consider the following space

$$
\Omega=\left\{y:(-\infty, b] \rightarrow \mathbb{R}:\left.y\right|_{(-\infty, 0]} \in \mathcal{B},\left.y\right|_{(0, b]} \in C_{1-\alpha}((0, b], \mathbb{R})\right\},
$$

where $\left.y\right|_{(0, b]}$ is the restriction of $y$ to $(0, b]$.

Definition 2.1 ([13]). Let $\alpha>0$ be a fixed number. The Riemann-Liouville fractional integral of order $\alpha$ with the lower limit zero for a function $\delta:(0, b] \rightarrow \mathbb{R}$ is determined as

$$
I_{0}^{\alpha} \delta(t)=\frac{1}{\Gamma(\alpha)} \int_{0}^{t}(t-s)^{\alpha-1} \delta(s) d s, \quad t \in(0, b],
$$

provided that the right-hand side is pointwisely defined on $(0, b]$, where $\Gamma($.$) is the gamma function.$

Definition 2.2 ([20]). Let $0<\alpha<1$. The Riemann-Liouville fractional derivative of order $\alpha$ with the lower limit zero for a function $\delta \in C((0, b], \mathbb{R})$ is characterized as

$$
D_{0}^{\alpha} \delta(t)=\frac{d}{d t} I_{0}^{1-\alpha} \delta(t), \quad t \in(0, b]
$$

Moreover, note that $D_{0}^{\alpha} t^{\alpha-1}=0$, and if $\delta \in L_{l o c}^{1}(0, b]$, then $D_{0}^{\alpha} I_{0}^{\alpha} \delta(t)=\delta(t)$.

Lemma 2.1 ([13]). Let $0<\alpha<1$. Then the unique solutions to the equation $D_{0}^{\alpha} \delta(t)=0$ are given by the formula $\delta(t)=C t^{\alpha-1}$, for $t>0$, where $C \in \mathbb{R}$ is a constant, provided $\delta \in$ $C(0, b] \cap L_{l o c}^{1}(0, b]$. Further, if $\delta \in C(0, b] \cap L_{l o c}^{1}(0, b]$ such that $D_{0}^{\alpha} \delta \in C(0, b] \cap L_{l o c}^{1}(0, b]$, then $I_{0}^{\alpha} D_{0}^{\alpha} \delta(t)=\delta(t)+C t^{\alpha-1}$ for $t>0$ and some constant $C \in \mathbb{R}$.

Definition 2.3. A function $y \in \Omega$ is said to be a solution of (5)-(6) if y satisfies the equation $D_{0}^{\alpha}\left[y(t)-g\left(t, \widetilde{y}_{t}\right)\right]=f\left(t, \widetilde{y}_{t}\right), t \in(0, b]$, with initial condition $\widetilde{y}_{0}=\phi,\left.y\right|_{(0, b]} \in C(0, b] \cap L_{l o c}^{1}(0, b]$ and $\frac{\partial g}{\partial t}$ exist.

In this paper, we consider the phase space $\left(\mathcal{B},\|\cdot\|_{\mathcal{B}}\right)$ which is a semi-normed linear space of functions mapping $(-\infty, 0]$ into $\mathbb{R}$, and fulfilling the following fundamental axioms that is similar to that introduced by Hale and Kato in [18] and widely discussed in [19]:

(H1) If $y:(-\infty, b] \rightarrow \mathbb{R}(b>0)$ is continuous on $(0, b]$ and $y_{0} \in \mathcal{B}$, then for every $t \in(0, b]$ the following conditions hold: 
(i) $y_{t} \in \mathcal{B}$;

(ii) $|y(t)| \leqslant H\left\|y_{t}\right\|_{\mathcal{B}}$, where $H>0$ is a constant;

(iii) $\left\|y_{t}\right\|_{\mathcal{B}} \leqslant K(t) \sup _{0 \leqslant s \leqslant t}|y(s)|+M(t)\left\|y_{0}\right\|_{\mathcal{B}}$, where $K, M:[0,+\infty) \rightarrow[0,+\infty)$ with $K$ continuous and $M$ locally bounded, such that $K, M$ are independent of $y($.$) . Denote$ $K_{b}=\sup \{K(t): t \in(0, b]\}$ and $M_{b}=\sup \{M(t): t \in(0, b]\}$.

(H2) For the function $y($.$) in (H1), the function t \rightarrow y_{t}$ is continuous from $(0, b]$ into $\mathcal{B}$.

(H3) The space $\mathcal{B}$ is complete.

Note that, the condition (H1)(ii) is equivalent to $|\phi(0)| \leqslant H\|\phi\|_{\mathcal{B}}$ for all $\phi \in \mathcal{B}$.

\section{Main results}

In this section, we exhibit and demonstrate the existence and uniqueness results for (5)-(6) under the Schauder fixed point theorem and the Banach fixed point theorem. Before starting and proving our main results, we assume that the following hypotheses are satisfied:

(A1) There exists a positive constant $L_{f}$ such that

$$
|f(t, u)-f(t, v)| \leqslant L_{f}\|u-v\|_{\mathcal{B}}, \quad t \in(0, b], \text { for any } u, v \in \mathcal{B} .
$$

(A2) There exists a positive constant $L_{g}$ such that

$$
|g(t, u)-g(t, v)| \leqslant L_{g}\|u-v\|_{\mathcal{B}}, \quad t \in(0, b], \text { for any } u, v \in \mathcal{B} .
$$

(A3) $f, g:(0, b] \times \mathcal{B} \rightarrow \mathbb{R}$ are continuous.

(A4) There exists a function $\eta \in L^{p}((0, b], \mathbb{R}), p>\frac{1}{\alpha}$ and a continuous non-decreasing function $\Psi:[0,+\infty) \rightarrow[0,+\infty)$ such that, for each $t \in(0, b]$, and every $u \in \mathcal{B}$

$$
|f(t, u)| \leqslant \eta(t) \Psi\left(\|u\|_{\mathcal{B}}\right) .
$$

(A5) The function $g$ is completely continuous and for any bounded set in $\Omega$, the set $\left\{t \rightarrow g\left(t, \widetilde{y}_{t}\right)\right.$ : $\widetilde{y} \in \mathcal{B}\}$ is equicontinuous in $C_{1-\alpha}((0, b], \mathbb{R})$ and there exist constants $0 \leqslant c_{1}<1, c_{2}>0$ such that, for $t \in(0, b]$ and $u \in \mathcal{B}$

$$
|g(t, u)| \leqslant c_{1}\|u\|_{\mathcal{B}}+c_{2} .
$$

Lemma 3.1. Let (A3) holds. The function $y \in \Omega$ is a solution of the fractional integral equation

$$
y(t)=t^{\alpha-1} \phi(0)+g\left(t, \widetilde{y}_{t}\right)+\frac{1}{\Gamma(\alpha)} \int_{0}^{t}(t-s)^{\alpha-1} f\left(s, \widetilde{y}_{s}\right) d s, \quad t \in(0, b]
$$

and $y(t)=\phi(t), t \in(-\infty, 0]$ if and only if $y$ is a solution of the fractional differential equation (5)-(6). provided that the integral in Eq. (8) exists. 
Proof. Applying the Riemann-Liouville fractional integral operator $I_{0}^{\alpha}$ on both sides of the for equation Eq. (5), we have

$$
I_{0}^{\alpha} D_{0}^{\alpha}\left[y(t)-g\left(t, \widetilde{y}_{t}\right)\right]=I_{0}^{\alpha} f\left(t, \widetilde{y}_{t}\right)
$$

In view of Lemma 2.1, we obtain

$$
y(t)=g\left(t, \widetilde{y}_{t}\right)+C t^{\alpha-1}+\frac{1}{\Gamma(\alpha)} \int_{0}^{t}(t-s)^{\alpha-1} f\left(s, \widetilde{y}_{s}\right) d s .
$$

Since $\widetilde{y}(t)=t^{1-\alpha} y(t)$ and use initial condition Eq. (6), then $\widetilde{y}(0)=C=\phi(0)$. Hence, the general solution to problem $(5)-(6)$ is

$$
y(t)=t^{\alpha-1} \phi(0)+g\left(t, \widetilde{y}_{t}\right)+\frac{1}{\Gamma(\alpha)} \int_{0}^{t}(t-s)^{\alpha-1} f\left(s, \widetilde{y}_{s}\right) d s, \text { for all } t \in(0, b] .
$$

Next, for $t \in(-\infty, 0], \widetilde{y}_{0}(t)=\phi(t)$ and $\phi(t)=\widetilde{\phi}(t)$. Thus by Eq. (7), we get

$$
y(t)=\phi(t), \quad t \in(-\infty, 0] .
$$

On the other hand, if Eq. (10) is satisfied for $t \in(0, b]$, then by using Definition 2.2, we have

$$
\begin{aligned}
D_{0}^{\alpha}\left[y(t)-g\left(t, \widetilde{y}_{t}\right)\right] & =D_{0}^{\alpha}\left(t^{\alpha-1} \phi(0)+g\left(t, \widetilde{y}_{t}\right)+\frac{1}{\Gamma(\alpha)} \int_{0}^{t}(t-s)^{\alpha-1} f\left(s, \widetilde{y}_{s}\right) d s-g\left(t, \widetilde{y}_{t}\right)\right)= \\
& =D_{0}^{\alpha}\left(t^{\alpha-1} \phi(0)+\frac{1}{\Gamma(\alpha)} \int_{0}^{t}(t-s)^{\alpha-1} f\left(s, \widetilde{y}_{s}\right) d s\right)= \\
& =D_{0}^{\alpha} t^{\alpha-1} \phi(0)+D_{0}^{\alpha} I_{0}^{\alpha} f\left(t, \widetilde{y}_{t}\right)= \\
& =D_{0}^{\alpha} I_{0}^{\alpha} f\left(t, \widetilde{y}_{t}\right)= \\
& =f\left(t, \widetilde{y}_{t}\right) .
\end{aligned}
$$

Finally, if Eq. (11) is satisfied, we have

$$
\widetilde{y}(s)=\widetilde{\phi}(s)=\phi(s), \forall s \in(-\infty, 0] .
$$

By using Eq. (7), we get

$$
\widetilde{y}(s)=\widetilde{y}_{0}(s), \forall s \in(-\infty, 0] .
$$

Comparing Eq. (12) and Eq. (13), we obtain $\widetilde{y}_{0}=\phi$, which completes the proof.

Our first result is based on the Banach fixed point theorem.

Theorem 3.1. If the hypotheses (A1), (A2) and (A3) hold, then there exists a unique solution to $(5)-(6)$ on $(-\infty, b]$ provided that

$$
\left(b^{1-\alpha} L_{g}+\frac{b}{\Gamma(\alpha+1)} L_{f}\right) K_{b}<1
$$

Proof. Transform the problem (5)-(6) into a fixed point problem. Consider the operator $N$ : $\Omega \rightarrow \Omega$ defined by

$$
(N y)(t)=\left\{\begin{array}{l}
t^{\alpha-1} \phi(0)+g\left(t, \widetilde{y}_{t}\right)+\frac{1}{\Gamma(\alpha)} \int_{0}^{t}(t-s)^{\alpha-1} f\left(s, \widetilde{y}_{s}\right) d s, \quad t \in(0, b] \\
\phi(t) \\
t \in(-\infty, 0]
\end{array}\right.
$$


For $\phi \in \mathcal{B}$, let $\widetilde{\phi}:(-\infty, b] \rightarrow \mathbb{R}$ be the extension of $\phi$ to $(-\infty, b]$ such that

$$
\widetilde{\phi}(t)=\left\{\begin{array}{l}
0, \quad t \in(0, b] \\
\phi(t), t \in(-\infty, 0]
\end{array}\right.
$$

Then $\widetilde{\phi}_{0}=\phi$. For each function $z \in C_{1-\alpha}((0, b], \mathbb{R})$ with $z(0)=0$, we consider the function $\widetilde{z}:(-\infty, b] \rightarrow \mathbb{R}$ be the extension of $z$ to $(-\infty, b]$ defined by

$$
\widetilde{z}(t)=\left\{\begin{array}{l}
t^{1-\alpha} z(t), \quad t \in(0, b] \\
0,
\end{array} \quad t \in(-\infty, 0] .\right.
$$

Then $\widetilde{z}_{0}=0$. If $y($.$) satisfies the integral equation$

$$
y(t)=t^{\alpha-1} \phi(0)+g\left(t, \widetilde{y}_{t}\right)+\frac{1}{\Gamma(\alpha)} \int_{0}^{t}(t-s)^{\alpha-1} f\left(s, \widetilde{y}_{s}\right) d s, \quad t \in(0, b],
$$

we can analyze $y(\cdot)$ as follows $y(t)=z(t)+\widetilde{\phi}(t), t \in(0, b]$, which implies that $\widetilde{y}_{t}=\widetilde{\phi}_{t}+\widetilde{z}_{t}$, for $t \in(0, b]$ and the function $z($.$) satisfies$

$$
z(t)=t^{\alpha-1} \phi(0)+g\left(t, \widetilde{\phi}_{t}+\widetilde{z}_{t}\right)+\frac{1}{\Gamma(\alpha)} \int_{0}^{t}(t-s)^{\alpha-1} f\left(s, \widetilde{\phi}_{s}+\widetilde{z}_{s}\right) d s, \quad t \in(0, b] .
$$

Set $\Omega_{0}=\left\{z \in \Omega, z_{0}=0\right\}$. For $z \in \Omega_{0}$, and let $\|\cdot\|_{\Omega_{0}}$ be seminorm in $\Omega_{0}$ described by

$$
\|z\|_{\Omega_{0}}=\left\|z_{0}\right\|_{\mathcal{B}}+\|z\|_{C_{1-\alpha}}=\sup \left\{\left|t^{1-\alpha} z(t)\right|: t \in(0, b]\right\} .
$$

Then $\left(\Omega_{0},\|z\|_{\Omega_{0}}\right)$ is a Banach space. Let the operator $T: \Omega_{0} \rightarrow \Omega_{0}$ be defined by

$$
(T z)(t)=z(t)=t^{\alpha-1} \phi(0)+g\left(t, \widetilde{\phi}_{t}+\widetilde{z}_{t}\right)+\frac{1}{\Gamma(\alpha)} \int_{0}^{t}(t-s)^{\alpha-1} f\left(s, \widetilde{\phi}_{s}+\widetilde{z}_{s}\right) d s, \quad t \in(0, b]
$$

and $(T z)_{0}=0$. Clearly, the fixed point of the operator $N$ is solution of the problem (5)-(6) and it's equivalent the fixed point of the operator $T$ that is also solution of Eq. (16). Now, we turn to proving that $T$ has a fixed point by means of the Banach fixed point theorem. For this purpose, it's enough to show that $T: \Omega_{0} \rightarrow \Omega_{0}$ is a contraction map. Indeed, by the hypothises, then for $z, z^{*} \in \Omega_{0}$ and for all $t \in(0, b]$, we have

$$
\begin{aligned}
& \left|t^{1-\alpha}(T z)(t)-t^{1-\alpha}\left(T z^{*}\right)(t)\right| \leqslant \\
\leqslant & t^{1-\alpha}\left|g\left(t, \widetilde{\phi}_{t}+\widetilde{z}_{t}\right)-g\left(t, \widetilde{\phi}_{t}+{\widetilde{z^{*}}}_{t}\right)\right|+ \\
& +\frac{t^{1-\alpha}}{\Gamma(\alpha)} \int_{0}^{t}(t-s)^{\alpha-1}\left|f\left(s, \widetilde{\phi}_{s}+\widetilde{z}_{s}\right)-f\left(s, \widetilde{\phi}_{s}+{\widetilde{z^{*}}}_{s}\right)\right| d s \leqslant \\
\leqslant & \left(b^{1-\alpha} L_{g}+\frac{b}{\Gamma(\alpha+1)} L_{f}\right)\left\|\widetilde{z}_{t}-{\widetilde{z^{*}}}_{t}\right\|_{\mathcal{B}} .
\end{aligned}
$$

Since

$$
\begin{aligned}
& \left\|\widetilde{z}_{t}-{\widetilde{z^{*}}}_{t}\right\|_{\mathcal{B}} \leqslant K(t) \sup _{0 \leqslant \tau \leqslant t}\left|\widetilde{z}(\tau)-\widetilde{z^{*}}(\tau)\right|+M(t)\left\|\widetilde{z}_{0}-{\widetilde{z^{*}}}_{0}\right\|_{\mathcal{B}} \leqslant \\
& \leqslant K_{b} \sup _{0 \leqslant \tau \leqslant t}\left|\tau^{1-\alpha} z(\tau)-\tau^{1-\alpha} z^{*}(\tau)\right| \leqslant \\
& \leqslant K_{b}\left\|z-z^{*}\right\|_{\Omega_{0}}
\end{aligned}
$$


we have

$$
\left|t^{1-\alpha}(T z)(t)-t^{1-\alpha}\left(T z^{*}\right)(t)\right| \leqslant\left(b^{1-\alpha} L_{g}+\frac{b}{\Gamma(\alpha+1)} L_{f}\right) K_{b}\left\|z-z^{*}\right\|_{\Omega_{0}}
$$

and hence

$$
\left\|T z-T z^{*}\right\|_{\Omega_{0}} \leqslant\left(b^{1-\alpha} L_{g}+\frac{b}{\Gamma(\alpha+1)} L_{f}\right) K_{b}\left\|z-z^{*}\right\|_{\Omega_{0}} .
$$

From inequality (14), then $T$ is contraction map in $\Omega_{0}$. As consequence of Banach fixed point theorem, we can conclude that $T$ has a unique fixed point which is just the unique solution to the integral equation Eq. (16) on $(0, b]$ that is

$$
z(t)=t^{\alpha-1} \phi(0)+g\left(t, \widetilde{\phi}_{t}+\widetilde{z}_{t}\right)+\frac{1}{\Gamma(\alpha)} \int_{0}^{t}(t-s)^{\alpha-1} f\left(s, \widetilde{\phi}_{s}+\widetilde{z}_{s}\right) d s, \quad t \in(0, b] .
$$

Set $y=\widetilde{\phi}+z$, then $y$ is the unique solution to the problem $(5)-(6)$ on $(-\infty, b]$.

Next, the following result gives an existence result based on the Schauder fixed point theorem.

Theorem 3.2. Assume that the hypotheses (A3), (A4) and (A5) hold. If

$$
K_{b}\left(c_{1} b^{1-\alpha}+\frac{b^{2}\|\eta\|_{p}}{\Gamma(\alpha+1)} \lim _{\zeta \rightarrow+\infty} \sup \frac{\Psi(\zeta)}{\zeta}\right)<1
$$

Then there exists at least a solution to (5)-(6) on $(-\infty, b]$.

Proof. Let the operator $T: \Omega_{0} \rightarrow \Omega_{0}$ be defined as in Eq. (18). We shall prove that the operator $T$ is continuous and completely continuous. To this end, we proceed in serval steps:

Step 1:T is continuous. Let $\left\{z_{n}\right\}_{n \in \mathbb{N}}$ be a sequence in $\Omega_{0}$ such that $z_{n} \rightarrow z$ in $\Omega_{0}$ as $n \rightarrow \infty$. Then for every $t \in(0, b]$, we have

$$
\begin{aligned}
& \left|t^{1-\alpha}\left(T z_{n}\right)(t)-t^{1-\alpha}(T z)(t)\right| \leqslant \\
& \leqslant t^{1-\alpha}\left|g\left(t, \widetilde{\phi}_{t}+\left(\widetilde{z}_{n}\right)_{t}\right)-g\left(t, \widetilde{\phi}_{t}+\widetilde{z}_{t}\right)\right|+ \\
& +\frac{t^{1-\alpha}}{\Gamma(\alpha)} \int_{0}^{t}(t-s)^{\alpha-1}\left|f\left(s, \widetilde{\phi}_{s}+\left(\widetilde{z}_{n}\right)_{s}\right)-f\left(s, \widetilde{\phi}_{s}+\widetilde{z}_{s}\right)\right| d s .
\end{aligned}
$$

From the continuity of $f$, the complete continuity of $g$ and the Lebesgue dominated convergence theorem, then $\left\|T z_{n}-T z\right\|_{\Omega_{0}} \rightarrow 0$ as $n \rightarrow \infty$. So $T$ is continuous.

Step 2: $T$ maps bounded subsets into bounded subsets in $\Omega_{0}$. Consider $\mathbb{B}_{r}=\left\{z \in \Omega_{0}\right.$ : $\left.\|z\|_{\Omega_{0}} \leqslant r\right\}$. By our hypothises, then for any $z \in \mathbb{B}_{r}$ and for each $t \in(0, b]$, we have

$$
\begin{aligned}
& \left|t^{1-\alpha}(T z)(t)\right| \leqslant|\phi(0)|+t^{1-\alpha}\left|g\left(t, \widetilde{\phi}_{t}+\widetilde{z}_{t}\right)\right|+\frac{t^{1-\alpha}}{\Gamma(\alpha)} \int_{0}^{t}(t-s)^{\alpha-1}\left|f\left(s, \widetilde{\phi}_{s}+\widetilde{z}_{s}\right)\right| d s \leqslant \\
& \leqslant H\|\phi\|_{\mathcal{B}}+t^{1-\alpha}\left(c_{1}\left\|\widetilde{\phi}_{t}+\widetilde{z}_{t}\right\|_{\mathcal{B}}+c_{2}\right)+\frac{t^{1-\alpha}}{\Gamma(\alpha)} \int_{0}^{t}(t-s)^{\alpha-1} \eta(s) \Psi\left(\left\|\widetilde{\phi}_{s}+\widetilde{z}_{s}\right\|_{\mathcal{B}}\right) d s .
\end{aligned}
$$

Since

$$
\begin{aligned}
&\left\|\widetilde{\phi}_{t}+\widetilde{z}_{t}\right\|_{\mathcal{B}} \leqslant\left\|\widetilde{\phi}_{t}\right\|_{\mathcal{B}}+\left\|\widetilde{z}_{t}\right\|_{\mathcal{B}} \leqslant \\
& \leqslant K(t) \sup _{0 \leqslant \tau \leqslant t}|\widetilde{\phi}(\tau)|+M(t)\left\|\widetilde{\phi}_{0}\right\|_{\mathcal{B}}+K(t) \sup _{0 \leqslant \tau \leqslant t}|\widetilde{z}(\tau)|+M(t)\left\|\widetilde{z}_{0}\right\|_{\mathcal{B}} \leqslant \\
&-541-
\end{aligned}
$$




$$
\begin{aligned}
& \leqslant M_{b}\|\phi\|_{\mathcal{B}}+K_{b} \sup _{0 \leqslant \tau \leqslant t}\left|\tau^{1-\alpha} z(\tau)\right|= \\
& =M_{b}\|\phi\|_{\mathcal{B}}+K_{b}\|z\|_{\Omega_{0}} \leqslant \\
& \leqslant M_{b}\|\phi\|_{\mathcal{B}}+K_{b} r \\
& :=r_{0}
\end{aligned}
$$

and use Hölder's inequality, then the inequality (22) becomes as follows

$$
\begin{aligned}
\left|t^{1-\alpha}(T z)(t)\right| & \leqslant H\|\phi\|_{\mathcal{B}}+t^{1-\alpha}\left(c_{1} r_{0}+c_{2}\right)+\frac{t^{1-\alpha}}{\Gamma(\alpha)} \Psi\left(r_{0}\right)\left(\int_{0}^{t}(t-s)^{(\alpha-1) q} d s\right)^{\frac{1}{q}}\|\eta\|_{p} \leqslant \\
& \leqslant H\|\phi\|_{\mathcal{B}}+b^{1-\alpha}\left(c_{1} r_{0}+c_{2}\right)+\frac{b^{2}\|\eta\|_{p}}{\Gamma(\alpha+1)} \Psi\left(r_{0}\right),
\end{aligned}
$$

where $q>1, \frac{1}{p}<\alpha, \frac{1}{p}+\frac{1}{q}=1$ and $\|\eta\|_{p}=\left(\int_{0}^{t}|\eta(s)|^{p} d s\right)^{\frac{1}{p}}$. Thus there exists a positive number

$$
\ell:=H\|\phi\|_{\mathcal{B}}+b^{1-\alpha}\left(c_{1} r_{0}+c_{2}\right)+\frac{b^{2}\|\eta\|_{p}}{\Gamma(\alpha+1)} \Psi\left(r_{0}\right),
$$

such that $\|T z\|_{\Omega_{0}} \leqslant \ell$. This implies that $T \mathbb{B}_{r} \subset \mathbb{B}_{\ell}$ i.e. $T$ maps bounded subsets into bounded subsets in $\Omega_{0}$.

Step 3: T maps bounded subsets into equicontinuous subsets in $\Omega_{0}$. Let $\mathbb{B}_{r}$ be a bounded set of $\Omega_{0}$ as in Step 2, and let also $z \in \mathbb{B}_{r}$ and for $t_{1}, t_{2} \in(0, b]$, with $0<t_{1}<t_{2} \leqslant b$, we have

$$
\begin{aligned}
& \left|t_{2}^{1-\alpha}(T z)\left(t_{2}\right)-t_{1}^{1-\alpha}(T z)\left(t_{1}\right)\right| \leqslant \\
\leqslant & \left|t_{2}^{1-\alpha} g\left(t_{2}, \widetilde{\phi}_{t_{2}}+\widetilde{z}_{t_{2}}\right)-t_{1}^{1-\alpha} g\left(t_{1}, \widetilde{\phi}_{t_{1}}+\widetilde{z}_{t_{1}}\right)\right|+ \\
& +\frac{t_{2}^{1-\alpha}-t_{1}^{1-\alpha}}{\Gamma(\alpha)} \int_{0}^{t_{2}}\left(t_{2}-s\right)^{\alpha-1}\left|f\left(s, \widetilde{\phi}_{s}+\widetilde{z}_{s}\right)\right| d s+ \\
& +\frac{t_{1}^{1-\alpha}}{\Gamma(\alpha)} \int_{0}^{t_{1}}\left|\left(t_{2}-s\right)^{\alpha-1}-\left(t_{1}-s\right)^{\alpha-1}\right|\left|f\left(s, \widetilde{\phi}_{s}+\widetilde{z}_{s}\right)\right| d s+ \\
& +\frac{t_{1}^{1-\alpha}}{\Gamma(\alpha)} \int_{t_{1}}^{t_{2}}\left(t_{2}-s\right)^{\alpha-1}\left|f\left(s, \widetilde{\phi}_{s}+\widetilde{z}_{s}\right)\right| d s .
\end{aligned}
$$

The complete continuity of $g$ imply that,

$$
\left|t_{2}^{1-\alpha} g\left(t_{2}, \widetilde{\phi}_{t_{2}}+\widetilde{z}_{t_{2}}\right)-t_{1}^{1-\alpha} g\left(t_{1}, \widetilde{\phi}_{t_{1}}+\widetilde{z}_{t_{1}}\right)\right| \rightarrow 0, \text { as } t_{1} \rightarrow t_{2},
$$

hence, from our hypothesis and Hölder's inequality, we obtain

$$
\begin{aligned}
& \left|t_{2}^{1-\alpha}(T z)\left(t_{2}\right)-t_{1}^{1-\alpha}(T z)\left(t_{1}\right)\right| \leqslant \\
\leqslant & \frac{t_{2}^{1-\alpha}-t_{1}^{1-\alpha}}{\Gamma(\alpha)} \int_{0}^{t_{2}}\left(t_{2}-s\right)^{\alpha-1} \eta(s) \Psi\left(\left\|\widetilde{\phi}_{s}+\widetilde{z}_{s}\right\|_{\mathcal{B}}\right) d s+ \\
& +\frac{t_{1}^{1-\alpha}}{\Gamma(\alpha)} \int_{0}^{t_{1}}\left(\left(t_{1}-s\right)^{\alpha-1}-\left(t_{2}-s\right)^{\alpha-1}\right) \eta(s) \Psi\left(\left\|\widetilde{\phi}_{s}+\widetilde{z}_{s}\right\|_{\mathcal{B}}\right) d s+ \\
& +\frac{t_{1}^{1-\alpha}}{\Gamma(\alpha)} \int_{t_{1}}^{t_{2}}\left(t_{2}-s\right)^{\alpha-1} \eta(s) \Psi\left(\left\|\widetilde{\phi}_{s}+\widetilde{z}_{s}\right\|_{\mathcal{B}}\right) d s \leqslant \\
\leqslant & \frac{t_{2}^{1-\alpha}-t_{1}^{1-\alpha}}{\Gamma(\alpha)} \Psi\left(r_{0}\right)\|\eta\|_{p}\left(\int_{0}^{t_{2}}\left(t_{2}-s\right)^{(\alpha-1) q} d s\right)^{\frac{1}{q}}+
\end{aligned}
$$




$$
\begin{aligned}
& +\frac{t_{1}^{1-\alpha}}{\Gamma(\alpha)} \Psi\left(r_{0}\right)\|\eta\|_{p}\left(\int_{0}^{t_{1}}\left(\left(t_{1}-s\right)^{(\alpha-1)}-\left(t_{2}-s\right)^{(\alpha-1)}\right)^{q} d s\right)^{\frac{1}{q}}+ \\
& +\frac{t_{1}^{1-\alpha}}{\Gamma(\alpha)} \Psi\left(r_{0}\right)\|\eta\|_{p}\left(\int_{t_{1}}^{t_{2}}\left(t_{2}-s\right)^{(\alpha-1) q} d s\right)^{\frac{1}{q}} \leqslant \\
& \leqslant \frac{\Psi\left(r_{0}\right)\|\eta\|_{p}}{e_{2} \Gamma(\alpha)}\left(b^{e_{1}}\left(t_{2}^{1-\alpha}-t_{1}^{1-\alpha}\right)+b^{1-\alpha}\left(\left(t_{1}^{e_{1}}-t_{2}^{e_{1}}\right)+2\left(t_{2}-t_{1}\right)^{e_{1}}\right)\right) \leqslant \\
& \leqslant \frac{\Psi\left(r_{0}\right)\|\eta\|_{p}}{e_{2} \Gamma(\alpha)}\left(b^{e_{1}}\left(t_{2}^{1-\alpha}-t_{1}^{1-\alpha}\right)+2 b^{1-\alpha}\left(t_{2}-t_{1}\right)^{e_{1}}\right),
\end{aligned}
$$

where $r_{0}$ be defined as in Step 2, $e_{1}=\frac{(\alpha-1) q+1}{q}$ and $e_{2}=((\alpha-1) q+1)^{\frac{1}{q}}>0$.

It follows that $\left|t_{2}^{1-\alpha}(T z)\left(t_{2}\right)-t_{1}^{1-\alpha}(T z)\left(t_{1}\right)\right| \rightarrow 0$, as $t_{1} \rightarrow t_{2}$, and the convergence is independent of $z$ in $\mathbb{B}_{r}$, which implies that the set $\left\{T \mathbb{B}_{r}\right\}$ is equicontinuous.

As a consequence of Steps 1-3, and along with the Arzela-Ascoli theorem, we can conclude that $T: \Omega_{0} \rightarrow \Omega_{0}$ is continuous and completely continuous.

To apply Schauder's fixed point theorem, we need to verify that there exists a closed convex bounded subset $\mathbb{B}_{\epsilon}=\left\{z \in \Omega_{0} ;\|z\|_{\Omega_{0}} \leqslant \epsilon\right\} \subseteq \Omega_{0}$ such that $T \mathbb{B}_{\epsilon} \subseteq \mathbb{B}_{\epsilon}$. For this purpose, it is clear that $\mathbb{B}_{\epsilon}$ is closed, convex and bounded of $\Omega_{0}$ for the each positive integer $\epsilon$, and we claim that there exists a positive integer $\epsilon$ such that $T \mathbb{B}_{\epsilon} \subseteq \mathbb{B}_{\epsilon}$. If this property is false, then for every positive integer $\epsilon$, there exists $z_{\epsilon} \in \mathbb{B}_{\epsilon}$ such that $\left(T z_{\epsilon}\right) \notin \mathbb{B}_{\epsilon}$, i.e. $\left\|T z_{\epsilon}(t)\right\|_{\Omega_{0}}>\epsilon$ for some $t_{\epsilon} \in(0, b]$ where $t_{\epsilon}$ denotes $t$ depending on $\epsilon$. However, by using the previous hypotheses, we have

$$
\begin{aligned}
\epsilon & <\left\|T z_{\epsilon}\right\|_{\Omega_{0}}=\sup _{0 \leqslant t \leqslant b}\left|t^{1-\alpha}\left(T z_{\epsilon}\right)(t)\right| \leqslant \\
& \leqslant \sup _{0 \leqslant t \leqslant b}\left\{|\phi(0)|+t^{1-\alpha}\left|g\left(t, \widetilde{\phi}_{t}+\left(\widetilde{z}_{\epsilon}\right)_{t}\right)\right|+\frac{t^{1-\alpha}}{\Gamma(\alpha)} \int_{0}^{t}(t-s)^{\alpha-1}\left|f\left(s, \widetilde{\phi}_{s}+\left(\widetilde{z}_{\epsilon}\right)_{s}\right)\right| d s\right\} \leqslant \\
& \leqslant \sup _{0 \leqslant t \leqslant b}\left\{\begin{array}{c}
H\|\phi\|_{\mathcal{B}}+t^{1-\alpha}\left(c_{1}\left\|\widetilde{\phi}_{t}+\left(\widetilde{z}_{\epsilon}\right)_{t}\right\|_{\mathcal{B}}+c_{2}\right) \\
+\frac{t^{1-\alpha}}{\Gamma(\alpha)} \int_{0}^{t}(t-s)^{\alpha-1} \eta(s) \Psi\left(\left\|\widetilde{\phi}_{s}+\left(\widetilde{z}_{\epsilon}\right)_{s}\right\|_{\mathcal{B}}\right) d s
\end{array}\right\}
\end{aligned}
$$

From Eq. (23), we can derive that $\left\|\widetilde{\phi}_{t}+\left(\widetilde{z}_{\epsilon}\right)_{t}\right\|_{\mathcal{B}} \leqslant M_{b}\|\phi\|_{\mathcal{B}}+K_{b} \epsilon:=\zeta$. This last inequality with Eq. (25) lead us to

$$
\zeta<M_{b}\|\phi\|_{\mathcal{B}}+K_{b}\left\{H\|\phi\|_{\mathcal{B}}+b^{1-\alpha}\left(c_{1} \zeta+c_{2}\right)+\frac{b^{2}\|\eta\|_{p}}{\Gamma(\alpha+1)} \Psi(\zeta)\right\} .
$$

Dividing both sides by $\zeta$ and taking the upper limit as $\zeta \rightarrow+\infty$, we get

$$
1<K_{b}\left(c_{1} b^{1-\alpha}+\frac{b^{2}\|\eta\|_{p}}{\Gamma(\alpha+1)} \lim _{\zeta \rightarrow+\infty} \sup \frac{\Psi(\zeta)}{\zeta}\right),
$$

which contradicts with Eq. (21). Hence, for some positive integer $\epsilon$, we must have $T \mathbb{B}_{\epsilon} \subseteq \mathbb{B}_{\epsilon}$.

An application of Schauder's fixed point theorem shows that there exists at least a fixed point $z$ of $T$ in $\Omega_{0}$. Then $y=z+\widetilde{\phi}$ is the solution to (5)-(6) on $(-\infty, b]$, and the proof is completed.

\section{Continuous dependence}

In this section, we discuss the influence of perturbed data to the solutions. 
Definition 4.1 ([13]). The solution $y \in C_{1-\alpha}((0, b])$ of the problem (5)-(6) is continuously dependent on initial data if for every $\phi, \psi \in \mathcal{B},\|y(\phi, .)-y(\psi, .)\|_{C_{1-\alpha}} \leqslant O\left(\|\phi-\psi\|_{\mathcal{B}}\right)$.

Definition 4.2 ([13]). The solution $y \in C_{1-\alpha}((0, b])$ of the problem (5)-(6) is continuously dependent on the two functions $f$ and $g$ if for every $f, \widetilde{f}, g, \widetilde{g} \in C((0, b] \times \mathcal{B}, \mathbb{R})$,

$$
\|y(g, f, .)-y(\widetilde{g}, \widetilde{f}, .)\|_{C_{1-\alpha}} \leqslant O(\sup |g-\widetilde{g}|)+O(\sup |f-\widetilde{f}|) .
$$

In our first result, we investigate at the continuous dependence of solutions on the initial values. The results previous remain valid if replace of the problem

$$
\begin{aligned}
& D_{0}^{\alpha}\left[y(t)-g\left(t, \widetilde{y}_{t}\right)\right]=f\left(t, \widetilde{y}_{t}\right), \quad t \in(0, b], \\
& \widetilde{y}_{0}=\psi \in \mathcal{B} .
\end{aligned}
$$

For that, we denote by $y(\phi,$.$) the solution to problem (5)-(6)$ and by $y(\psi,$.$) the solution to$ problem (26)-(27).

Theorem 4.1. Assume that the hypotheses of Theorem 3.1 are satisfied. If

$$
\left(b^{1-\alpha} L_{g}+\frac{b}{\Gamma(\alpha+1)} L_{f}\right) K_{b}<1,
$$

then there exists a constant $\kappa$ such that

$$
\|y(\phi, .)-y(\psi, .)\|_{C_{1-\alpha}} \leqslant \kappa\|\phi-\psi\|_{\mathcal{B}}, \quad \forall \phi, \psi \in \mathcal{B} .
$$

Proof. In view of Theorem 3.1, we know that for every $\phi, \psi \in \mathcal{B}$, the equation Eq. (5) has solutions $y(\phi,$.$) and y(\psi,$.$) on (-\infty, b]$, respectively. Further, there are $z_{1}, z_{2} \in C_{1-\alpha}((0, b])$ such that $y(\phi,)=.z_{1}+\widetilde{\phi}$, and $y(\psi,)=.z_{2}+\widetilde{\psi}$. Then $z_{1}$ and $z_{2}$ satisfy

$$
\begin{aligned}
& z_{1}(t)=t^{\alpha-1} \phi(0)+g\left(t, \widetilde{\phi}_{t}+\left(\widetilde{z_{1}}\right)_{t}\right)+\frac{1}{\Gamma(\alpha)} \int_{0}^{t}(t-s)^{\alpha-1} f\left(s, \widetilde{\phi}_{s}+\left(\widetilde{z_{1}}\right)_{s}\right) d s, \quad t \in(0, b] . \\
& z_{2}(t)=t^{\alpha-1} \psi(0)+g\left(t, \widetilde{\psi}_{t}+\left(\widetilde{z_{2}}\right)_{t}\right)+\frac{1}{\Gamma(\alpha)} \int_{0}^{t}(t-s)^{\alpha-1} f\left(s, \widetilde{\psi}_{s}+\left(\widetilde{z_{2}}\right)_{s}\right) d s, \quad t \in(0, b] .
\end{aligned}
$$

and $y(\phi, t)=z_{1}(t), y(\psi, t)=z_{2}(t)$ for $t \in(0, b]$. Hence for all $t \in(0, b]$, we have

$$
\begin{aligned}
& \left|t^{1-\alpha} y(\phi, t)-t^{1-\alpha} y(\psi, t)\right|=\left|t^{1-\alpha} z_{1}(t)-t^{1-\alpha} z_{2}(t)\right| \leqslant \\
& \leqslant|\phi(0)-\psi(0)|+t^{1-\alpha}\left|g\left(t, \widetilde{\phi}_{t}+\left(\widetilde{z_{1}}\right)_{t}\right)-g\left(t, \widetilde{\psi_{t}}+\left(\widetilde{z_{2}}\right)_{t}\right)\right|+ \\
& +\frac{t^{1-\alpha}}{\Gamma(\alpha)} \int_{0}^{t}(t-s)^{\alpha-1}\left|f\left(s, \widetilde{\phi}_{s}+\left(\widetilde{z_{1}}\right)_{s}\right)-f\left(s, \widetilde{\psi}_{s}+\left(\widetilde{z_{2}}\right)_{s}\right)\right| d s \leqslant \\
& \leqslant H\|\phi-\psi\|_{\mathcal{B}}+t^{1-\alpha} L_{g}\left(\left\|\widetilde{\phi}_{t}-\widetilde{\psi}_{t}\right\|_{\mathcal{B}}+\left\|\left(\widetilde{z_{1}}\right)_{t}-\left(\widetilde{z_{2}}\right)_{t}\right\|_{\mathcal{B}}\right)+ \\
& +\frac{t^{1-\alpha}}{\Gamma(\alpha)} \int_{0}^{t}(t-s)^{\alpha-1} L_{f}\left(\left\|\widetilde{\phi}_{s}-\widetilde{\psi_{s}}\right\|_{\mathcal{B}}+\left\|\left(\widetilde{z_{1}}\right)_{s}-\left(\widetilde{z_{2}}\right)_{s}\right\|_{\mathcal{B}}\right) d s \leqslant \\
& \leqslant\left(\frac{H}{M_{b}}+b^{1-\alpha} L_{g}+\frac{b}{\Gamma(\alpha+1)} L_{f}\right) M_{b}\|\phi-\psi\|_{\mathcal{B}}+\left(b^{1-\alpha} L_{g}+\frac{b}{\Gamma(\alpha+1)} L_{f}\right) K_{b}\left\|z_{1}-z_{2}\right\|_{C_{1-\alpha}} . \\
& \text { Since }\left(b^{1-\alpha} L_{g}+\frac{b}{\Gamma(\alpha+1)} L_{f}\right) K_{b}<1 \text {, then } \\
& \|y(\phi, .)-y(\psi, .)\|_{C_{1-\alpha}} \leqslant\left(\frac{H}{M_{b}}+b^{1-\alpha} L_{g}+\frac{b}{\Gamma(\alpha+1)} L_{f}\right) M_{b}\|\phi-\psi\|_{\mathcal{B}}+
\end{aligned}
$$




$$
\begin{aligned}
& +\left(b^{1-\alpha} L_{g}+\frac{b}{\Gamma(\alpha+1)} L_{f}\right) K_{b}\|y(\phi, .)-y(\psi, .)\|_{C_{1-\alpha}} \leqslant \\
\leqslant & \frac{\left(\frac{H}{M_{b}}+b^{1-\alpha} L_{g}+\frac{b}{\Gamma(\alpha+1)} L_{f}\right) M_{b}}{1-\left(b^{1-\alpha} L_{g}+\frac{b}{\Gamma(\alpha+1)} L_{f}\right) K_{b}}\|\phi-\psi\|_{\mathcal{B}} .
\end{aligned}
$$

Take, $\kappa=\frac{\left(\frac{H}{M_{b}}+b^{1-\alpha} L_{g}+\frac{b}{\Gamma(\alpha+1)} L_{f}\right) M_{b}}{1-\left(b^{1-\alpha} L_{g}+\frac{b}{\Gamma(\alpha+1)} L_{f}\right) K_{b}}$, we get

$$
\|y(\phi, .)-y(\psi, .)\|_{C_{1-\alpha}} \leqslant \kappa\|\phi-\psi\|_{\mathcal{B}} .
$$

Next, we study the continuous dependence of solutions on the given functions $f$ and $g$. Now, we denote by $y(g, f,$.$) the solution to problem (5)-(6)$ and by $y(\widetilde{g}, \widetilde{f},$.$) the solution to the$ problem

$$
\begin{aligned}
& D_{0}^{\alpha}\left[y(t)-\widetilde{g}\left(t, \widetilde{y}_{t}\right)\right]=\widetilde{f}\left(t, \widetilde{y}_{t}\right), \quad t \in(0, b], \\
& \widetilde{y}_{0}=\phi \in \mathcal{B} .
\end{aligned}
$$

Theorem 4.2. Let $f, \widetilde{f}, g$ and $\widetilde{g}$ fulfill hypotheses (A1), (A2) and (A3). If

$$
\left(b^{1-\alpha} L_{g}+\frac{b}{\Gamma(\alpha+1)} L_{f}\right) K_{b}<1,
$$

then there exist two constants $\kappa_{1}$ and $\kappa_{2}$ such that

$\|y(g, f, .)-y(\widetilde{g}, \widetilde{f}, .)\|_{C_{1-\alpha}} \leqslant \kappa_{1} \sup _{(t, u) \in(0, b] \times \mathcal{B}}|g(t, u)-\widetilde{g}(t, u)|+\kappa_{2} \sup _{(t, u) \in(0, b] \times \mathcal{B}}|f(t, u)-\widetilde{f}(t, u)|$.

Proof. The existence and uniqueness results can be confirmed by Theorem 3.1 and Theorem 3.2. Let $z_{1}, z_{2} \in C_{1-\alpha}((0, b])$ be such that $y(g, f,)=.z_{1}+\widetilde{\phi}$, and $y(\widetilde{g}, \widetilde{f},)=.z_{2}+\widetilde{\phi}$. Then $z_{1}$ and $z_{2}$ satisfy

$$
z_{1}(t)=t^{\alpha-1} \phi(0)+g\left(t, \widetilde{\phi}_{t}+\left(\widetilde{z_{1}}\right)_{t}\right)+\frac{1}{\Gamma(\alpha)} \int_{0}^{t}(t-s)^{\alpha-1} f\left(s, \widetilde{\phi}_{s}+\left(\widetilde{z_{1}}\right)_{s}\right) d s, \quad t \in(0, b] .
$$

and

$$
z_{2}(t)=t^{\alpha-1} \phi(0)+\widetilde{g}\left(t, \widetilde{\phi}_{t}+\left(\widetilde{z_{2}}\right)_{t}\right)+\frac{1}{\Gamma(\alpha)} \int_{0}^{t}(t-s) \widetilde{f}\left(s, \widetilde{\phi}_{s}+\left(\widetilde{z_{2}}\right)_{s}\right) d s, \quad t \in(0, b] .
$$

Consequently, for $t \in(0, b]$, we have

$$
\begin{aligned}
& \left|t^{1-\alpha} y(g, f, t)-t^{1-\alpha} y(\widetilde{g}, \widetilde{f}, t)\right|= \\
= & \left|t^{1-\alpha} z_{1}(t)-t^{1-\alpha} z_{2}(t)\right| \leqslant \\
\leqslant & t^{1-\alpha}\left|g\left(t, \widetilde{\phi}_{t}+\left(\widetilde{z_{1}}\right)_{t}\right)-g\left(t, \widetilde{\phi}_{t}+\left(\widetilde{z_{2}}\right)_{t}\right)\right|+t^{1-\alpha}\left|g\left(t, \widetilde{\phi}_{t}+\left(\widetilde{z_{2}}\right)_{t}\right)-\widetilde{g}\left(t, \widetilde{\phi}_{t}+\left(\widetilde{z_{2}}\right)_{t}\right)\right|+ \\
& +\frac{t^{1-\alpha}}{\Gamma(\alpha)} \int_{0}^{t}(t-s)^{\alpha-1}\left|f\left(s, \widetilde{\phi}_{s}+\left(\widetilde{z_{1}}\right)_{s}\right)-f\left(s, \widetilde{\phi}_{s}+\left(\widetilde{z_{2}}\right)_{s}\right)\right| d s+ \\
& +\frac{t^{1-\alpha}}{\Gamma(\alpha)} \int_{0}^{t}(t-s)^{\alpha-1}\left|f\left(s, \widetilde{\phi}_{s}+\left(\widetilde{z_{2}}\right)_{s}\right)-\widetilde{f}\left(s, \widetilde{\phi}_{s}+\left(\widetilde{z_{2}}\right)_{s}\right)\right| d s \leqslant
\end{aligned}
$$




$$
\begin{aligned}
\leqslant & t^{1-\alpha} L_{g}\left\|\left(\widetilde{z_{1}}\right)_{t}-\left(\widetilde{z_{2}}\right)_{t}\right\|_{\mathcal{B}}+t^{1-\alpha} \sup _{(t, u) \in(0, b] \times \mathcal{B}}|g(t, u)-\widetilde{g}(t, u)|+ \\
& +\frac{t}{\Gamma(\alpha+1)} L_{f} \|\left(\widetilde{\left.z_{1}\right)_{s}}-\left(\widetilde{z_{2}}\right)_{s} \|_{\mathcal{B}}+\frac{t}{\Gamma(\alpha+1)} \sup _{(t, u) \in(0, b] \times \mathcal{B}}|f(t, u)-\widetilde{f}(t, u)| \leqslant\right. \\
\leqslant & \left(b^{1-\alpha} L_{g}+\frac{b}{\Gamma(\alpha+1)} L_{f}\right) K_{b}\left\|z_{1}-z_{2}\right\|_{C_{1-\alpha}}+b^{1-\alpha} \sup _{(t, u) \in(0, b] \times \mathcal{B}}|g(t, u)-\widetilde{g}(t, u)|+ \\
& +\frac{b}{\Gamma(\alpha+1)} \sup _{(t, u) \in(0, b] \times \mathcal{B}}|f(t, u)-\widetilde{f}(t, u)| .
\end{aligned}
$$

Therefore, since $\left(b^{1-\alpha} L_{g}+\frac{b}{\Gamma(\alpha+1)} L_{f}\right) K_{b}<1$, we have

$$
\begin{aligned}
& \|y(g, f, .)-y(\widetilde{g}, \widetilde{f}, .)\|_{C_{1-\alpha}} \leqslant \\
\leqslant & \left(b^{1-\alpha} L_{g}+\frac{b}{\Gamma(\alpha+1)} L_{f}\right) K_{b}\|y(g, f, .)-y(\widetilde{g}, \widetilde{f}, .)\|_{C_{1-\alpha}}+ \\
& +b^{1-\alpha} \sup _{(t, u) \in(0, b] \times \mathcal{B}}|g(t, u)-\widetilde{g}(t, u)|+\frac{b}{\Gamma(\alpha+1)} \sup _{(t, u) \in(0, b] \times \mathcal{B}}|f(t, u)-\widetilde{f}(t, u)| \leqslant \\
\leqslant & \frac{b^{1-\alpha}}{1-\left(b^{1-\alpha} L_{g}+\frac{b}{\Gamma(\alpha+1)} L_{f}\right) K_{b}(t, u) \in(0, b] \times \mathcal{B}}|g(t, u)-\widetilde{g}(t, u)|+ \\
& +\frac{b}{\Gamma(\alpha+1)\left(1-\left(b^{1-\alpha} L_{g}+\frac{b}{\Gamma(\alpha+1)} L_{f}\right) K_{b}\right)} \sup _{(t, u) \in(0, b] \times \mathcal{B}}|f(t, u)-\widetilde{f}(t, u)| .
\end{aligned}
$$

Let $\kappa_{1}=\frac{b^{1-\alpha}}{1-\left(b^{1-\alpha} L_{g}+\frac{b}{\Gamma(\alpha+1)} L_{f}\right) K_{b}}$ and $\kappa_{2}=\frac{b}{\Gamma(\alpha+1)\left(1-\left(b^{1-\alpha} L_{g}+\frac{b}{\Gamma(\alpha+1)} L_{f}\right) K_{b}\right)}$.

Then we obtain

$$
\begin{aligned}
& \|y(g, f, .)-y(\widetilde{g}, \widetilde{f}, .)\|_{C_{1-\alpha}} \leqslant \\
\leqslant & \kappa_{1} \sup _{(t, u) \in(0, b] \times \mathcal{B}}|g(t, u)-\widetilde{g}(t, u)|+\kappa_{2} \sup _{(t, u) \in(0, b] \times \mathcal{B}}|f(t, u)-\widetilde{f}(t, u)| .
\end{aligned}
$$

\section{Remark 4.1.}

1. The problem (5)-(6) with $g \equiv 0$ has been discussed by Dong [14]. The author proved the existence and continuous dependence of solutions for (3)-(4) with nonzero initial values in a weighted space of continuous functions when $f(t, u)$ satisfies the Lipschitz condition with respect to $u$. He also studied existence results when $f(t, u)$ does not satisfy the Lipschitz condition considering the condition $f$ under $L^{p}$ space. His proof was by means of Banach contraction principle and Schauder's theorem.

2. Kilbas et al. in [21], have investigated local existence and uniqueness results for the same problem with $g \equiv 0$ in the space of summable functions. And they also considered $f(t, u)$ satisfies the Lipschitz condition with respect to $u$. The technique of their proof was based on the method of the successive approximation.

3. In this paper, we have made some development on Dong's work [14]. In addition $g \neq 0$. The development is done by using the discretization technique through Banach fixed point theorem and Schauder fixed point theorem. Moreover, the problem that is considered here is different from that has been considered by Dong in [14]. 


\section{Conclusion}

We have successfully developed appropriate conditions for existence and uniqueness of solutions to a weighted neutral functional differential equation (5)-(6) with fractional order and infinite delay. The respective conditions have been derived by using fixed point theorem of Banach and Schauder type. Moreover, we also discussed the influence of perturbed data of solutions in weighted space $C_{1-\alpha}(0, b]$.

The paper did make some development on Dong's work [14]. In [14], he studied the existence and continuous dependence for weighted functional fractional differential equation (3)-(4) with infinite delay. The development was done by using the discretization technique. Moreover the problem that is considered here is different from that has been considered by Dong in [14].

The authors thanks the editor and referees for their careful reading of the manuscript and insightful comments, which helped to improve the quality of the paper. The authors would also like to acknowledge the valuable comments and suggestions from the editors, which vastly contributed to the improvement of the presentation of the paper.

\section{References}

[1] S.Abbas, M.Benchohra, G.M.N'Guérékata, Topics in fractional differential equations, Springer Science, Business Media, 2012.

[2] M.S.Abdo, S.K.Panchal, Effect of perturbation in the solution of fractional neutral functional differential equations, J. KSIAM, 22(2018), no. 1, 63-74.

[3] M.S.Abdo, S.K.Panchal, Existence and continuous dependence for fractional neutral functional differential equations, J. Mathematical Model., 5(2017), no. 2, 153-170.

[4] R.Agarwal, Y.Zhou, Y.He, Existence of fractional neutral functional differential equations, Compu. Math. Applic., 59(2010), no. 3, 1095-1100.

[5] O.A.Arino, T.A.Burton, J.R.Haddock, Periodic solutions to functional differential equations, Proceedings Roy. Soc. Edinb. Sec. A Math., 101(1985), no. 3-4, 253-271.

[6] A.Belarbi, M.Benchohra, A.Ouahab, Uniqueness results for fractional functional differential equations with infinite delay in Fréchet spaces, Applicable Anal., 85(2006), no. 12, 1459.

[7] M.Benchohra, J.Henderson, S.K.Ntouyas, A.Ouahab Existence results for fractional order functional differential equations with infinite delay, J. Math. Anal. Appl., 338 (2008), no. 2, $1340-1350$.

[8] J.Cao, H.Chen, W.Yang, Existence and continuous dependence of mild solutions for fractional neutral abstract evolution equations, Adv. Differ. Equ., 2015(2015), no. 1, 1-6.

[9] D.Delboso, L.Rodino, Existence and uniqueness for a nonlinear fractional differential equation, J. Math. Anal. Appl., 204(1996), 609-625.

[10] J.Deng, Q.Hailiang, New uniqueness results of solutions for fractional differential equations with infinite delay, Compu. Math. Appl., 60(2010), no. 8, 2253-2259. 
[11] K.Diethelm, N.J.Ford, Analysis of fractional differential equations, J. Math. Anal. Appl., 265(2002), 229-248.

[12] K.Diethelm, G.Walz, Numerical solution of fractional order differential equations by extrapolation, Numer. Algorithms, 16(1997), 231-253.

[13] K.Diethelm, The Analysis of Fractional Differential Equations, Lecture Notes in Mathematics 2004, Springer, Berlin, 2010.

[14] Q.Dong, Existence and continuous dependence for weighted fractional differential equations with infinite delay, Adv. in Differe. Equ., 2014(2014), no. 1, 1-13.

[15] K.M.Furati, N.E.Tatar, An existence result for a nonlocal fractional differential problem, J. Fract. Calc., 26(2004), 4351.

[16] L.Gaul, P.Klein, S.Kempfle, Damping description involving fractional operators, Mech. Sys. Sign. Processing, 5(1991), 81-88.

[17] W.G.Glockle, T.F.Nonnenmacher, A fractional calculus approach of self-similar protein dynamics, Biophys. J., 68(1995), 46-53.

[18] J.Hale, J.Kato, Phase space for retarded equations with infinite delay, Funkcial. Ekvac., 21(1978), 11-41.

[19] Y.Hino, S.Murakami, T.Naito, Functional differential equations with infinite delay, Lecture Notes in Math, vol. 1473, Springer-Verlag, Berlin, 1991.

[20] A.A.Kilbas, S.A.Marzan, Nonlinear differential equations with the Caputo fractional derivative in the space of continuously differentiable functions, Differ. Uravn., 41(2005), no. 1, $82-86$.

[21] A.A.Kilbas, H.M.Srivastava, J.J.Trujillo, Theory and Applications of Fractional Differential Equations, North-Holland Math. Stud., vol. 204, Elsevier, Amsterdam, 2006.

[22] V.Lakshmikantham, S.Leela, J.V.Devi, Theory of fractional dynamic systems, Cambridge Scientific Publishers, 2009.

[23] V.Lakshmikantham, Theory of fractional functional differential equations, Nonlinear Anal. Th. Meth. Appl., 69(2008), no. 10, 3337-3343.

[24] F.Metzler, W.Schick, H.G.Kilian, T.F.Nonnenmacher, Relaxation in filled polymers: A fractional calculus approach, J. Chem. Phys., 103(1995), 7180-7186.

[25] K.S.Miller, B.Ross, An Introduction to the Fractional Calculus and Differential Equations, John Wiley, New York, 1993.

[26] S.M.Momani, S.B.Hadid, Z.M.Alawenh, Some analytical properties of solutions of differential equations of noninteger order, Int. J. Math. Sci., 2004(2004), 697-701.

[27] S.M.Momani, S.B.Hadid, Some comparison results for integro-fractional differential inequalities, J. Fract. Calc., 24(2003), 37-44.

[28] I.Podlubny, Fractional Differential Equations, Academic Press, San Diego, 1999. 
[29] S.G.Samko, A.A.Kilbas, O.I.Marichev, Fractional Integrals and Derivatives. Theory and Applications, Gordon and Breach, Yverdon, 1993.

[30] C.Yu, G.Gao, Existence of fractional differential equations, J. Math. Anal. Appl., 310(2005), $26-29$.

\section{Взвешенные дробные нейтральные функционально-дифференциальные уравнения}

Мохаммед С. Абдо

Департамент математики Маратвада университет Аурангавад, 431004 Индия

Департамент математики

Ходейда университет Аль-Ходейда, 3114

Йемен

Сатиш К. Паншал

Департамент математики Маратвада университет Аурангавад, 431004

Индия

В настоящей работе рассматривается взвешенное нейтральное функциональнодифференциальное уравнение дробного порлдка $0<\alpha<1$ с ненулевыми начальными значениями, бесконечное замедление и стандартная дробная производная Римана-Лиувилля. Используя ряд инструментов дробного исчисления, включая теорему Шаудера о неподвижной точке и теорему Банаха о неподвижной точке, проверяем существование, единственность и непрерывную зависимость решения взвешенной нейтральной задачи.

Ключевые слова: дробные функиионально-дифференииальные уравнения, дробная производная и дробный интеграл, существование и непрерывная зависимость, теорема о неподвижной точке. 